\title{
Classification of cow's behaviors based on 3-DoF accelerations from cow's movements
}

\author{
Phung Cong Phi Khanh ${ }^{1}$, Kieu Thi Nguyen ${ }^{2}$, Nguyen Dinh-Chinh ${ }^{3}$, Tran Duc-Nghia ${ }^{4}$, \\ Hoang Quang Trung ${ }^{5}$, Nguyen Van Thang ${ }^{6}$, Tran Duc-Tan ${ }^{7}$ \\ ${ }^{1,3,7}$ Electronics and Telecommunication Faculty, VNU, Hanoi - University of Engineering and Technology, Viet Nam \\ ${ }^{1}$ Hanoi National University of Education, Viet Nam \\ ${ }^{2}$ Faculty of Mechanical, Electrical, and Electronic Engineering, Nguyen Tat Thanh University, Viet Nam \\ ${ }^{4}$ Institute of Information Technology, Vietnamese Academy of Science and Technology, Viet Nam \\ ${ }^{5}$ University of Information and Communication Technology, Viet Nam \\ ${ }^{6}$ Thuy Loi University, Viet Nam
}

\section{Article Info}

Article history:

Received Jul 30, 2018

Revised Dec 18, 2018

Accepted Dec 29, 2018

Keywords:

Acceleration

Behaviors

Classification

Sensors

\begin{abstract}
Cow's behavior classification helps people to monitor cow activities, thus the health and physiological periods of cows can be well tracked. To classify the behavior of cows, the data from the 3-axis acceleration sensor mounted on their neck is often used. Data acquisition and preprocessing of sensor data is required in this device. We acquire data from the 3-axis acceleration sensor mounted on the cows'neck and send to the microcontrollter. At the microcontroller, a proposed decision tree is applied in real-time manner to classify four important activities of the cows (standing, lying, feeding, and walking). Finally, the results can be sent to the server through the wireless transmission module. The test results confirm the reliability of the proposed device.
\end{abstract}

Copyright (C) 2019 Institute of Advanced Engineering and Science. All rights reserved.

\section{Corresponding Author:}

Tran Duc-Tan,

Electronics and Telecommunication Faculty,

VNU, Hanoi-University of Engineering and Technology,

E3 building, 144 Xuan Thuy, Cau Giay, Ha Noi, Viet Nam.

Email: tantd@vnu.edu.vn

\section{INTRODUCTION}

Demand for clean beef and cow's milk on the world in general and VIETNAM in particular is increasing. To meet that demand, dairy farming requires a high technique and a large investment [1]. However, farmers encountered a lot of difficulties because of small, scattered breeding, unskilled, full households and using traditional breeding methods. This is the main reason made to a high in the costs of production. Therefore, dairy breeding needs to apply modern technology in the world to improve productivity and product quality [2]. Aware of the importance of this issue, some of milk processing companies in Vietnam that implement advanced technology such as TH true milk applying Israel's technology (AFIMILK).

In the world, most of monitoring of systems is based on accelerometer data of sensor [3], [4]. The reason is that acceleration data can reflect how fast an object changes its velocity [5], [6]. To classify behavior, we need to choose a suitable machine-learning algorithm. Complex machine learning may offer a good result of classification [7], [8], [9]. However, the computation would be large which cannot be embedded to the device [10]. The device needs to send the acceleration data to the server, and classification algorithm must be installed and run on the server.

In the paper, we choose the decision tree algorithm to classify behavioral state of cow because we can embed the classification process in the device (we do not need to send the acceleration data to the server). Moreover, it also offers other advantages such as automatic feature selection, little data prep effort, handles 
data non-linearity, easy to interpret. We consider four important activities of cows: standing, lying, feeding and walking.

\section{SYSTEM IMPLEMENTATION}

Figure 1 shows a block diagram of the analyzing system. There are two data streams in this system: the first stream is acceleration data from MPU6050 sensor [11] and the second is the video recorded cow's activities from a camera. Data from these streams are then synchronized for the performance evaluation.

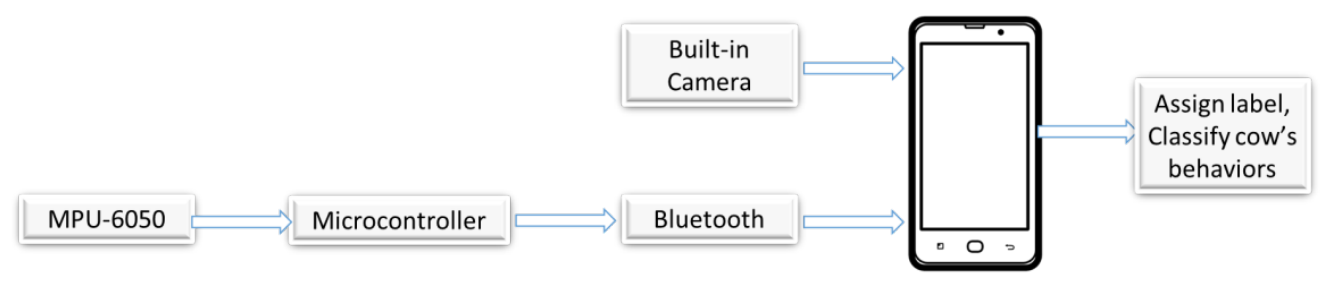

Figure 1. Block diagram of the analyzing system

Figure 2 shows the device that acquires the acceleration data from the cow's neck. MPU6050 is used to sense the acceleration from the cow's movement, and send to the microcontroller PIC18F45K20 [12]. PIC18F45K20 send the data to a mobile phone via a Bluetooth module. The camera that recorded the cow's activities is also from built-in camera of this mobile phone. The device is quite compact and light, thus, it is suitable for the cow to wear. After the analyzing procedure, a classifying algorithm will be embedded into PIC18F45K20 in order to provide the real-time status of the cow (standing, lying, feeding, and walking).

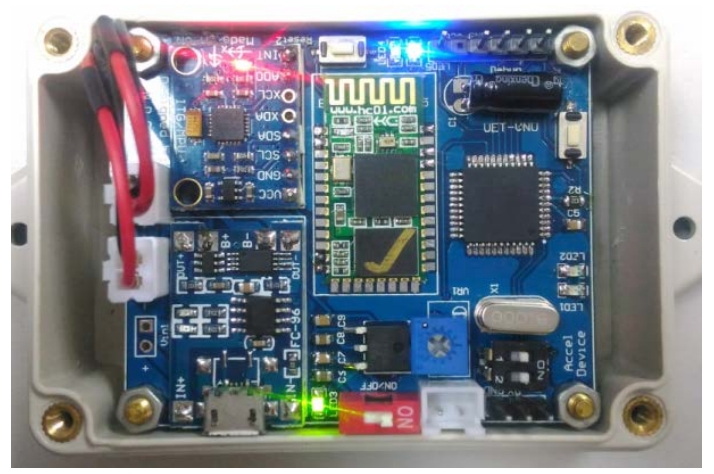

Figure 2. A photo of the device

\subsection{Data processing}

Firstly, the acceleration signal is brought to a low pass filter (LPF) to smooth the acceleration data. The acceleration data can be divided into the static and the dynamic components. The static component of the acceleration is caused by the orientation of the sensor relative to the gravity field of the earth which determine the body posture. We extract the static acceleration component for each axis $\mu_{i, t}(\mathrm{i}=\mathrm{X}, \mathrm{Y}, \mathrm{Z})$ by using a running mean filter:

$$
\mu_{i, t}=\frac{1}{\text { Win_size }} \sum_{\frac{t-W i n \_s i z e}{2}}^{\frac{t+\text { Win_size }}{2}} A_{i t}^{*}
$$

where $\mathrm{t}$ is time index, win_size is the window size, and $A_{i, t}^{*}$ is the acceleration data, respectively. The dynamic component of the acceleration Ai,t is caused directly by the movement of the cow. 


$$
\mathrm{Ai}, \mathrm{t}=\left|A_{i, t}^{*}-\mu_{i, t}\right|
$$

The L2-norm of Ax,t , Ay,t , and Az,t provide us

$$
A_{\text {norm }, t}=\sqrt{A_{x, t}^{2}+A_{y, t}^{2}+A_{z, t}^{2}}
$$

In this study, we use two features $\mu_{y, t}$ and $A_{\text {norm, },}$ to classsify the cow's behavior.

\subsection{Flowchart}

Four different states of the cow are classified by using the flowchart as shown in Figure 3. This is a decision tree algorithm where two features $\mu_{y, t}$ and $A_{\text {norm, } t}$ are applied. $A_{\text {norm, } t}$ is used to classify between the high and the low dynamic activities. If the cow is in the high dynamic, the mean of $\mu_{y, t}$ is applied to classify between the feeding and the walking status. In the case of a low activity, we compare the values of $\mu_{y, t}$. To predefine optimal thresholds B1, B2 for classifying the standing and lying status.

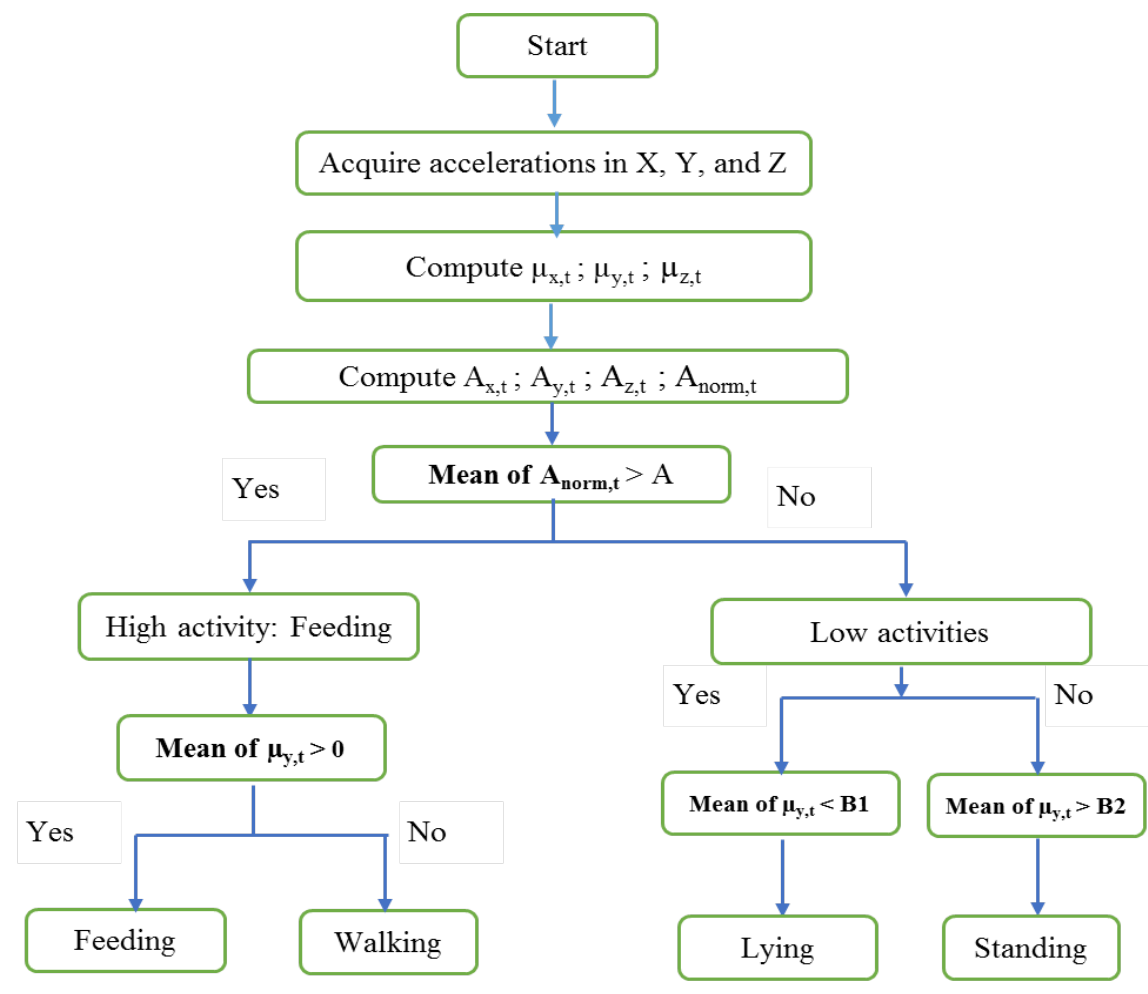

Figure 3. Decision tree algorithm flowchart

\section{RESULTS AND DISCUSSION}

Figure 4 shows the position of the device on the cow's neck. The device has small size, light weights, and it's comforting to the cow. Figure 5 presents the samples of acceleration $A_{x, t}^{*} A_{y, t}^{*} A_{z, t}^{*}$ acquired from the experiments which includes standing, lying, feeding and walking activities. We can see that when the cow is standing or lying, the signal on each axis have changed a little because both behaviors show small movement. If the cow is fed or walking, its movement is larger. Specifically, head of the cow will continuously shake left, right. Thus, we can clearly observe the change of acceleration. There is also the large difference in the component of y-axis between lying and standing, feeding and walking.

Figure 6 shown two features $\mu_{y, t}$ and $A_{n o r m, t}$ which corresponds to the acceleration data in Figure 5. We see that the value of $A_{\text {norm,t }}$ in standing and lying of state is smaller than in feeding and walking activities. The values of $\mu_{y, t}$ in feeding and walking are clearly different, the value of $\mu_{y, t}$ in feeding is larger than $0 \mathrm{mg}$ while in walking is smaller than $0 \mathrm{mg}$ ). Therefore, we can classify quite accurate behaviors of cows by choosing optimal threshold values. 


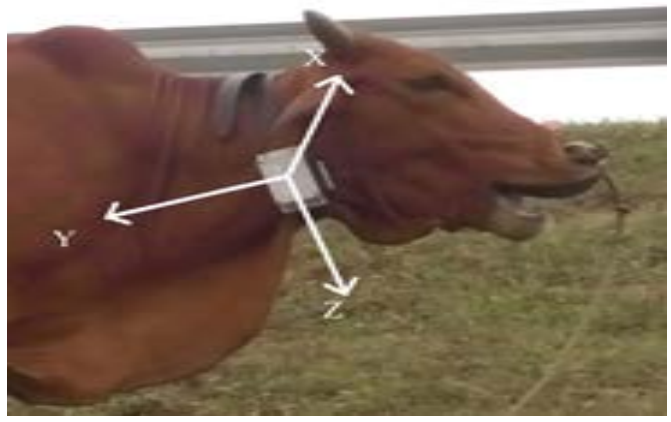

Figure 4. A cow is wearing the device on its neck

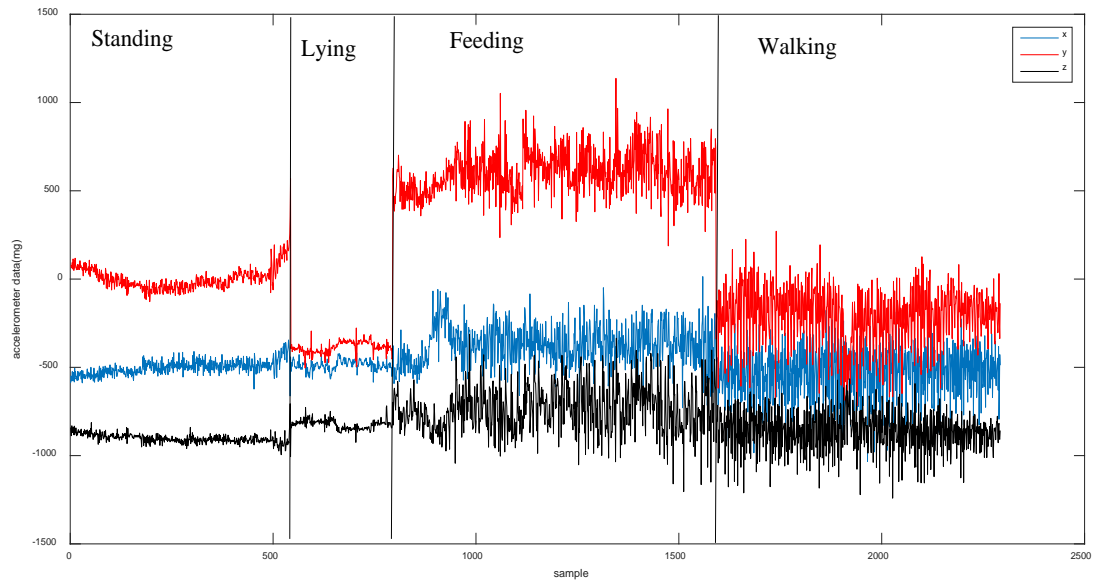

Figure 5. Experimental acceleration data in X, Y, Z axes

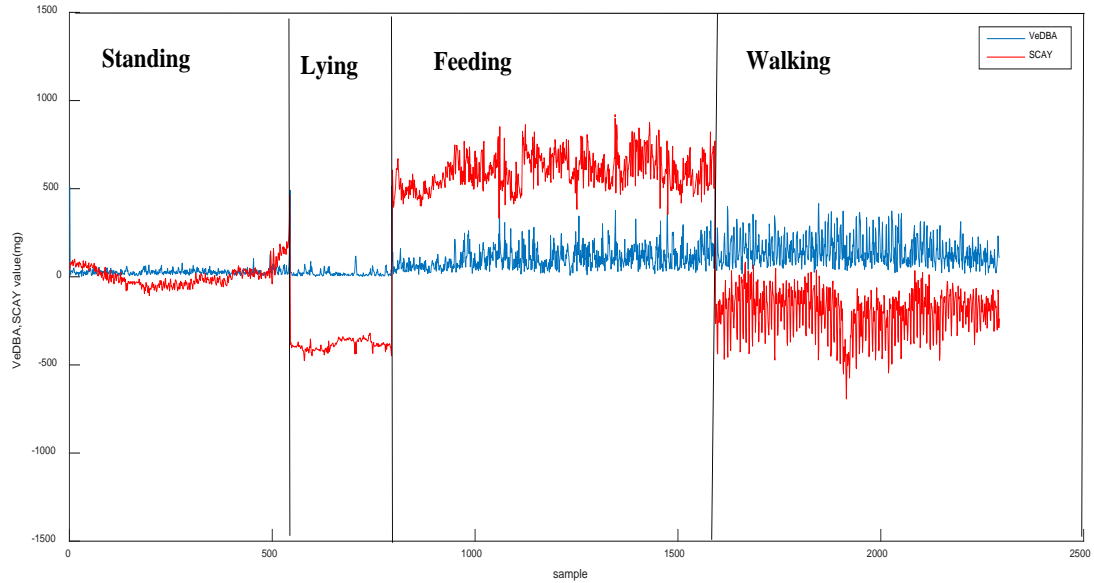

Figure 6. VeDBA, SCAY value under four activities

Three thresholds A, B1 and B2 determine the classification performance. Thus, we need to find these optimal values for these thresholds [13], [14]. In this study, ROC curve is used to choose A, B1 and B2. Figure 7 shows the ROC curve obtained with the resolution of $1 \mathrm{mg}$ ( $1 \mathrm{mg}=10-3 \mathrm{~g})$. The optimal threshold value is the one that generates a pair of TPR and FPR value closest to the top left corner that represents the value that separates perfectly the positives from the negatives. The square points are chosen for determining A, B1 and B2. We found threshold A, B1, B2 are 48mg, -133mg, -108mg, respectively. 

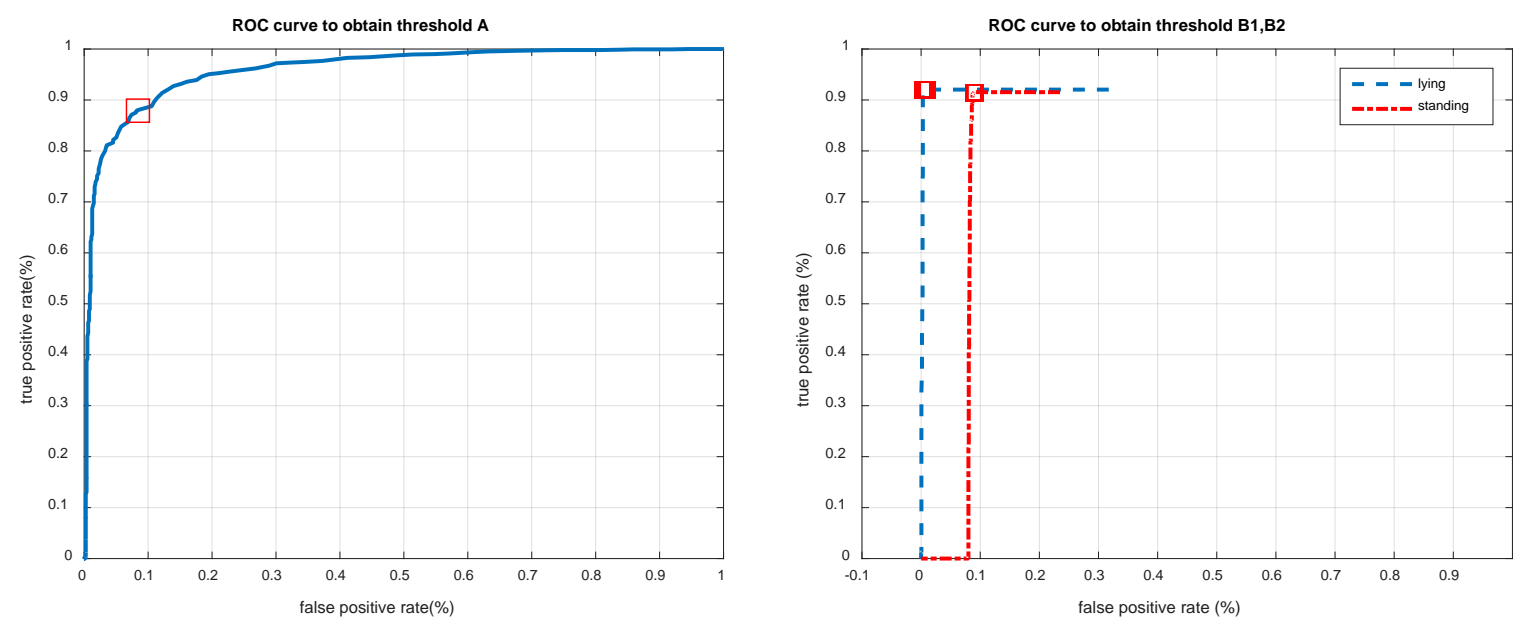

Figure 7. ROC curve to obtain threshold A, B1, B2

The system's performance of our system is shown in the Table 1. For overall performance corresponding to this dataset, sensitivity, precision and specificity are $99 \%, 91.02 \%$, and $75.08 \%$, respectively. Note that, we can compromise among these factors by adjusting three thresholds (A, B1, and B2). By using the decision tree algorithm, this work can classify 4 different activities of cows (in [15], the authors only concerned to classify 3 activities which are standing, lying, and feeding).

Table 1. Performance Evaluation of the Classification

\begin{tabular}{cccc}
\hline State & Sensitivity (\%) & Precision (\%) & Specificity (\%) \\
\hline Standing & 100 & 76 & 61.8 \\
Lying & 100 & 90 & 96.3 \\
Feeding & 100 & 94 & 94.4 \\
Walking & 97 & 93.6 & 93.9 \\
Overall & 99 & 88.5 & 86.7 \\
\hline
\end{tabular}

\section{CONCLUSION}

In this paper, we have successfully built a device that is able to classify four important activities of cows (standing, lying, feeding, and walking). This device can work in real-time manner because it used the simple and effective decision tree algorithm. It offers a good result of classification such as the sensitivity of $99 \%$, the specificity of $86.7 \%$, and the precision of $88.5 \%$. In the decision tree algorithm, we used three thresholds A, B1, B2 to classify the cow's activities. It also provides a flexible scheme to adjust the performance indexes and customize the most suitable thresholds for each cow (e.g. small or big cows). The device has small size, and light weights (i.e. under $0.3 \mathrm{~kg}$ ). Therefore, it can apply to real farms. In the future, we will continue to trains more cow to wear our devices, enhance the classification performance by combining the decision tree algorithm with SVM algorithm, and classify more behaviors such as cripple, evolution, etc. Moreover, we will develop a solution to help to farm owner always to follow the situations of cows while sitting in their room or travelling.

\section{ACKNOWLEDGEMENTS}

This work was supported by University of Information and Communication Technology (ICTU), Thai Nguyen University, code HĐ2018-TN07-02.

\section{REFERENCES}

[1] Phillips, C., 2008. Cattle behaviour and welfare. John Wiley \& Sons.

[2] Baratchi, M., Meratnia, N., Havinga, P. J., Skidmore, A. K., \& Toxopeus, B. A. "Sensing solutions for collecting spatio-temporal data for wildlife monitoring applications: a review," Sensors, Vol. 13, No. 5, pp. 6054-6088, 2013. 
[3] Gian Quoc Anh, Nguyen Dinh Chinh, Tran Duc Nghia, Tran Duc-Tan, "Monitoring of Landslides in Mountainous Regions based on FEM Modelling and Rain Gauge Measurements,“ International Journal of Electrical and Computer Engineering (IJECE), ISSN: 2088-8708, Vol. 6, No. 5, pp. 2106-2113, 2016.

[4] Vimalkumar, K., Vinodhini, R. E., \& Archanaa, R. “An early detection-warning system to identify speed breakers and bumpy roads using sensors in smartphones,” International Journal of Electrical and Computer Engineering (IJECE), 7(3), 1377-1384, 2017.

[5] Qasem, L, Cardew, A, Wilson, A, Griffiths, I, Halsey, LG, Shepard, ELC, Gleiss, AC \& Wilson, 'Tri-axial dynamic acceleration as a proxy for animal energy expenditure; should we be summing values or calculating the vector?” 2012 .

[6] Chen, K. Y., \& DAVID R BASSETT, J. R. "The technology of accelerometry-based activity monitors: current and future,” Medicine \& Science in Sports \& Exercise, Vol. 37, No.11, pp. S490-S500, 2005.

[7] Mannini, A., \& Sabatini, A. M. "Machine learning methods for classifying human physical activity from on-body accelerometers,” Sensors, Vol. 10, No. 2, pp.1154-1175, 2010.

[8] Martiskainen, P., Järvinen, M., Skön, J. P., Tiirikainen, J., Kolehmainen, M., \& Mononen, J. "Cow behaviour pattern recognition using a three-dimensional accelerometer and support vector machines,” Applied animal behaviour science, Vol. 119, No. 1-2, pp. 32-38, 2009.

[9] Hoang, Q.-T., Phi Khanh, P. C., Trung Ninh, B., Phuong Dung, C. T., \& Tran, T. “Cow Behavior Monitoring Using a Multidimensional Acceleration Sensor and Multiclass SVM,” International Journal of Machine Learning and Networked Collaborative Engineering, 2(03), pp. 110-118, 2018.

[10] Patil, R., \& Tamane, S. "A Comparative Analysis on the Evaluation of Classification Algorithms in the Prediction of Diabetes,” International Journal of Electrical and Computer Engineering (IJECE), 8(5), 2018.

[11] Fedorov, D. S., A. Yu Ivoilov, V. A. Zhmud, and V. G. Trubin. "Using of Measuring System MPU6050 for the Determination of the Angular Velocities and Linear Accelerations," Automatics \& Software Enginery 11, no. 1, 75-80, 2015.

[12] Mikhaylov, Konstantin, and Jouni Tervonen. "Evaluation of power efficiency for digital serial interfaces of microcontrollers," In 2012 5th International Conference on New Technologies, Mobility and Security (NTMS), pp. 1-5. IEEE, 2012.

[13] Saxena, Abhinav, et al. "Metrics for evaluating performance of prognostic techniques," International conference on Prognostics and Health management, pp. 1-17, 2008.

[14] Resheff, Yehezkel S., et al. "AcceleRater: a web application for supervised learning of behavioral modes from acceleration measurements,” in Movement ecology, Vol. 2, No.1, pp. 1-7, 2014.

[15] Khanh, P. C. P., Long, T. T., Chinh, N. D., \& Duc-Tan, T. "Performance evaluation of a multi-stage classification for cow behavior.” In Recent Advances in Signal Processing, Telecommunications \& Computing (SigTelCom), 2018 2nd International Conference on IEEE, (pp. 121-125), January 2018.

\section{BIOGRAPHIES OF AUTHORS}
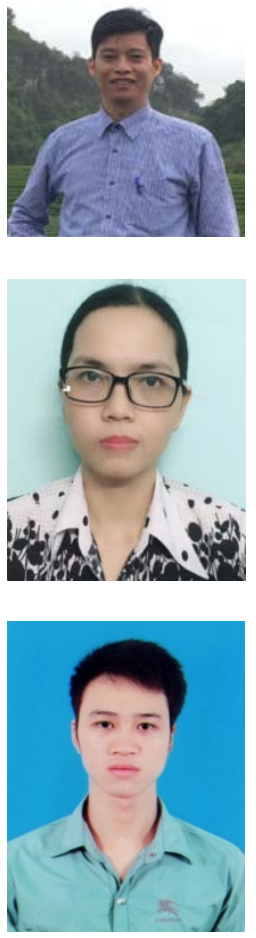

Phung Cong Phi-Khanh received the B.S. degree in Technology Education, Hanoi National University of Education in 1998 and M.S. degree in Electronic and Communication from VNU University of Engineering and Technology (VNU-UET) in 2005. He is currently Phd. student at VNU-UET. His present research interests are in digital signal processing and embedded systems.

Kieu Thi Nguyen was born in 1983. She is a head department of mechanical, Faculty of Mechanical, Electrical, and Electronic Engineering, Nguyen Tat Thanh University, Vietnam. She did her master at HCMC University of Technology and Education, Vietnam. Her research interests are network and signal processing.

Nguyen Dinh-Chinh received the B.S. degree in Electronic and Telecommunication from Vietnam National University, Hanoi - University of Engineering and Technology in 2014 and M.S. degree in Electronic and Communication from the same university in 2017. His research interests are digital signal processing, machine learning and embedded systems. 


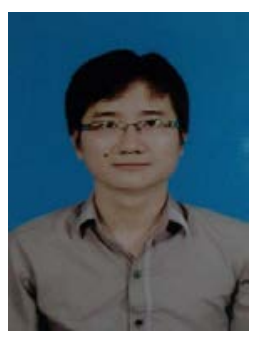

Tran Duc-Nghia was born in 1986. He is a scientist at Institute of Information Technology (IOIT), Vietnam Academy of Science and Technology (VAST). He is currently a PHD student of 'Drug, Toxicology, Chemistry, Imageries' (MTCI ED 563) doctoral school of Sorbonne Paris Cité (France). His research interests are mathematics and signal processing, Electron Paramagnetic Resonance (EPR), parameter estimation, data analysis. In his thesis, he focuses on signal processing of EPR spectra for in vivo experiments. He did his master in 'Science in Information Technology' at University of Engineering and Technology, VNU.

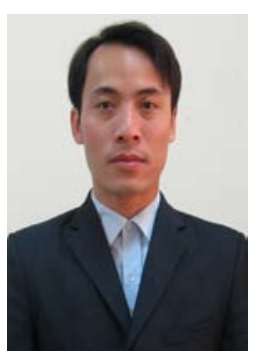

Hoang Quang-Trung received the B.Sc. and M.Sc. degrees from the Vietnam National University, and the Ph.D. degree in electronics engineering from the Le Quy Don Technique University, Vietnam, in 2017. He is currently working as a lecturer at Thai Nguyen University of Information and Communication Technology, Vietnam. His research interests include digital communications and wireless networks.

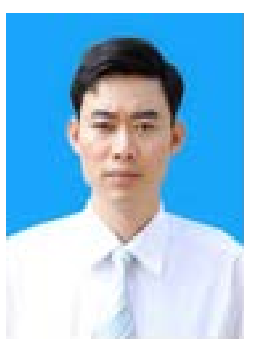

Nguyen Van Thang was born in 1979. He received his B.Sc., degree in Electronics and Telecommunication at the Hanoi University of Transport and Communications, Hanoi, Vietnam, in 2002 and his M.Sc. degree in Information Engineering from Le Quy Don University, Hanoi, Vietnam, in 2007. He received his PhD. degree in Electronics and Telecommunication at VNU University of Engineering and Technology. Now, he is lecturer at ThuyLoi University. Until now, he is author and coauthor of some papers on MEMS based sensors and their application.

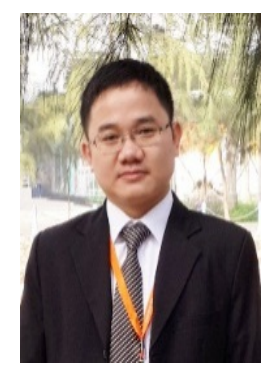

Tran Duc-Tan was born in 1980. He received his B.Sc, M.Sc, and PhD. degrees respectively in 2002, 2005, and 2010 at the University of Engineering and Technology (UET), Vietnam National University-Hanoi, Vietnam (VNUH), where he has been a lecturer since 2006. He was the recipient of the Vietnam National University, Hanoi, Vietnam Young Scientific Award in 2008. He is currently an Associate Professor with the Faculty of Electronics and Telecommunications, University of Engineering and Technology, Vietnam National University, Hanoi, Vietnam. He is the author and coauthor of 50 papers on MEMS based sensors and their application. His present research interest is in DSP applications. 